\title{
USO DE MEMBRANA AMNIÓTICA COMO COBERTURA TEMPORAL EN PACIENTES CON QUEMADURAS DEL HOSPITAL LUIS VERNAZA
}

\author{
USE OF AMNIOTIC MEMBRANE AS TEMPORARY COVERAGE IN BURN PATIENTS AT THE LUIS VERNAZA
}

HOSPITAL

\author{
UTILIZAÇÃO DE MEMBRANA AMNIOTICA COMO COBERTURA TEMPORÁRIA EM PACIENTES COM \\ QUEIMADURAS DO HOSPITAL LUIS VERNAZA
}

\author{
FERNANDO QUINTANA JEDERMANN ${ }^{1,2}$, STALIN DE LOOR ZAMBRANO ${ }^{1,2}$ \\ 1 Universidad Católica de Santiago de Guayaquil, Guayaquil, Ecuador \\ ${ }^{2}$ Hospital Luis Vernaza, Guayaquil, Ecuador
}

Resumen

El paciente con quemaduras demanda de un manejo complejo, y el uso de membrana amniótica como cobertura temporal conforma un método muy valioso para favorecer su evolución. Este método forma parte de las alternativas terapéuticas en la Unidad de Quemados del hospital Luis Vernaza (HLV). Objetivos: demostrar la eficiencia del amnios humano como cobertura temporal en pacientes con quemaduras. Metodología: se presenta una serie de 14 casos de pacientes con quemaduras de menos del $25 \%$ de superficie corporal total, tratados en la Unidad de Quemados del HLV, donde se aplicó membrana amniótica humana. Resultados: el $100 \%$ de los pacientes requirió solo de una aplicación, 9 pacientes fueron del sexo masculino ( $64 \%$ ) y 5 del femenino ( $36 \%$ ), con un rango de edades entre 25 y 86 años. La media de edad fue 45 años y mediana de 47 años, la causa de lesión más frecuente fue a causa de flama con 9 casos ( $64 \%$ ). El promedio de estancia hospitalaria postoperatoria fue de 9 días, con un límite inferior de 6 y superior de 13. En ningún paciente se observó signos de infección o rechazo inmunológico y en todos se observó re-epitelización de las lesiones. En la escala de valoración numérica del dolor (del 1 al 10), se confirmó que todos los pacientes referían menos dolor y posteriormente al día 7 desapareció en todos. Discusión: el uso de membrana amniótica humana en pacientes quemados evita la pérdida de líquidos, electrolitos y proteínas en las áreas cruentas, a modo de barrera artificial ayuda a prevenir las infecciones especialmente, como una membrana basal sustituta, sin desmerecer la importancia del efecto analgésico sobre las lesiones.

PALABRAS CLAVE: quemaduras, amnios, cicatrización de heridas.

Abstract

Burn patients demand complex treatment and the use of amniotic membrane as temporary coverage forms a valuable method to favor their evolution. This method is part of the therapeutic alternatives in the Burn Unit of the Luis Vernaza Hospital (LVH). Objectives: to demonstrate the efficiency of human amnion as a temporary coverage in burn patients. Methodology: this research presents a series of 14 cases of patients with burns of less than $25 \%$ of the total body surface that were treated at the Burn Unit of LVH, where human amniotic membrane was applied. Results: $100 \%$ of patients required only one application, 9 patients were male (64\%) and 5 female (36\%), with an age range between 25 and 86 . The mean age was 45 and the median was 47 , the most common cause of injury was due to flame with 9 cases (64\%). The average postoperative hospital stay was 9 days, with a lower limit of 6 and an upper limit of 13 . No signs of infection or immune rejection were observed in any of the patients and all re-epithelialization of lesions was observed in all of them. On the pain scale (from 1 to 10), it was confirmed that all the patients reported less pain and later it completely disappeared on the 7th day. Discussion: the use of human amniotic membrane in burn patients prevents loss of fluids, electrolytes and proteins in the bloody areas, as an artificial barrier it especially helps prevent infections like a substitute basement membrane, without detracting from the importance of the analgesic effect on injuries.

KEYWORDS: burns, amnion, wound healing.

Resumo

0 paciente com queimaduras precisa de gestão complexa e 0 uso de membrana amniótica como cobertura temporária constitui um método valioso para favorecer a sua evolução. Este método é parte de alternativas terapêuticas na Unidade de Queimados do Hospital Luis Vernaza (HLV). Objetivos: demonstrar a eficiência do âmnio humano como cobertura temporária em pacientes com queimaduras. Metodologia: apresenta uma série de 14 casos de pacientes com queimaduras de menos de $25 \%$ da superfície corporal total, tratados na unidade de queimados do HLV, onde a membrana amniótica humana foi aplicada. Resultados: $100 \%$ dos pacientes necessitaram de apenas uma aplicação, nove pacientes foram do sexo masculino (64\%) e cinco (5) do sexo feminino (36 $\%$ ), com um faixa de idades entre 25 e 86 anos. A média de idade foi de 45 anos e mediana de 47 anos, a causa de lesão mais frequente foi a chama com 9 $\operatorname{casos}(64 \%$ ). A média de internação pós-operatória foi de nove dias, com limite inferior de seis e maiores de 13 . Em nenhum paciente foi observado sinais de infecção ou rejeição imune e em todos foi observado re-epitelização das lesões. Na escala de valoração numérica da dor (1 a 10), foi confirmado que todos os pacientes tinham menos dor e posteriormente ao dia sete (7) desapareceu. Discussão: 0 uso de membrana amniótica humana em pacientes queimados impede a perda de fluidos, eletrólitos e proteínas nas áreas cruentas, a maneira de barreira artificial ajuda a prevenir infecções em particular como uma substituição da membrana basal, sem desmerecer a importância do efeito analgésico sobre as lesões.

PALABRAS-CHAVE: queimaduras, âmnio, cicatrização. 
INTRODUCCIÓN

El amnios humano es una membrana fetal unida al corion constituyendo el saco amniótico que, lleno de líquido amniótico, brinda protección al feto y confiere un medio adecuado para su crecimiento. Es una membrana fina, semi transparente (figura 1), compuesta por una capa interna epitelial, una gruesa membrana basal y una capa estromal avascular de tejido conectivo fetal con escasa cantidad de fibroblastos'. Anatómicamente, la membrana amniótica humana (MAH) tiene 5 capas: 1 ) epitelio, 2) membrana basal, 3) capa compacta, 4) capa fibroblástica y 5) una capa esponjosa. Las últimas tres capas están compuestas de matriz del estroma. ${ }^{2,3}$

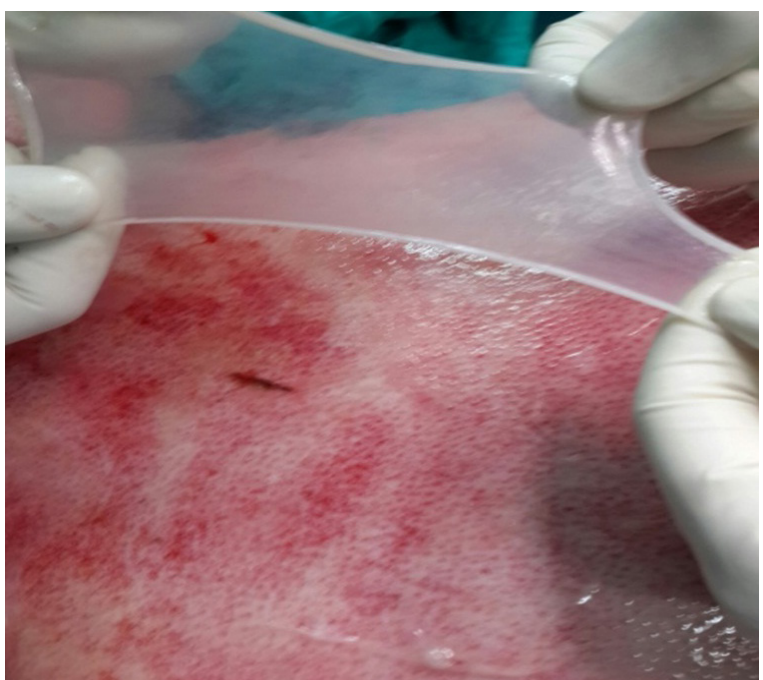

Figura 1. Aplicación de membrana amniótica en herida posterior a quemadura por flama en tórax anterior.

El uso de la membrana amniótica, fue puesto en práctica por el Dr. Davis en 1910, como trasplante de piel. ${ }^{4}$ En 1913, Stern y Sabella al aplicar membrana amniótica sobre ulceraciones y quemaduras de la superficie cutánea observaron que no se producía infección de las heridas, había reducción del dolor y aumentó la velocidad de reepitelización. ${ }^{5,6}$ Roth en 1940 , sin resultados muy buenos, fue el primero en emplear membrana amniótica en enfermedades de superficie ocular. ${ }^{7}$ Luego Sorsby y Col usaron la amnioplastía, MAH procesada químicamente, como recubrimiento temporal en tratamiento de quemaduras oculares. En 1995, Kim y Tseng, comunicaron el éxito de la MA preservada como sustrato para reconstruir córneas de conejo con deficiencia de hemocitoblastos límbicos. ${ }^{8}$ Posteriormente el interés por su uso en oftalmología tuvo más acogida, y en la actualidad una gran lista de patologías de esa índole, incluyendo quemaduras químicas de superficie ocular, son tratadas con este medio.?

Diversos estudios han establecido que la MAH regula el transporte hidroelectrolítico, disminuye el crecimiento bacteriano, presen-ta escasa inmunogenicidad, facilita la reepitelización, alivia el dolor, disminuye la inflamación e inhibe la apoptosis de las células epiteliales; además evita la vascularización y la fibrosis de la córnea, lo que la hace útil en el uso de heridas de superficie ocular. ${ }^{9-11} \mathrm{El}$ uso de membrana amniótica constituye una excelente alternativa en el tratamiento de quemaduras, en las que se necesita reconstruir superficies cruentas. Debido a sus propiedades permite su uso en diversas patologías. En la capa estromal existen factores liberadores, de proliferación y angiogénicos, que reducen la inflamación y modulan la cicatrización. ${ }^{12}$ Esta $\mathrm{MAH}$, asimismo, expresa el factor de crecimiento epidérmico (EGF), factor de crecimiento del hepatocito y factor de crecimiento del queratinocito. ${ }^{12,13}$ La capa epitelial compuesta por un epitelio cúbico simple se asienta sobre una gruesa membrana basal rica en colágenos III y IV y laminina favorece la adhesión y migración celular, y por tal motivo estimula la reepitelización propiedad muy valorada (figura 2a, 2b y 3a, 3b). Otra propiedad de gran importancia es la ausencia de reacción inmune ante este tejido, evitando de esta manera una reacción inflamatoria. ${ }^{4}$

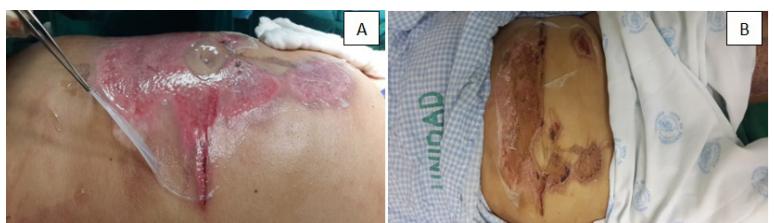

Figura 2A. Paciente femenino con quemaduras en región toracoabdominal por líquido caliente mientras se aplica amnios. B. Postoperatorio de 9 días.

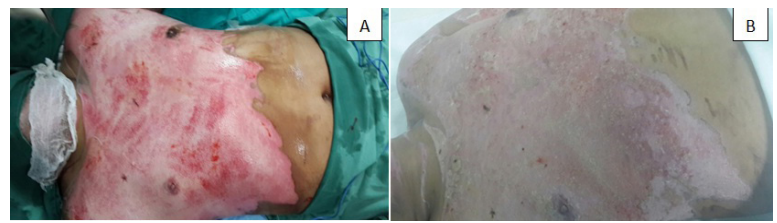

Figura 3A. Preoperatorio de paciente masculino con quemaduras en tronco anterior. B. Postoperatorio de 9 días con membrana amniótica.
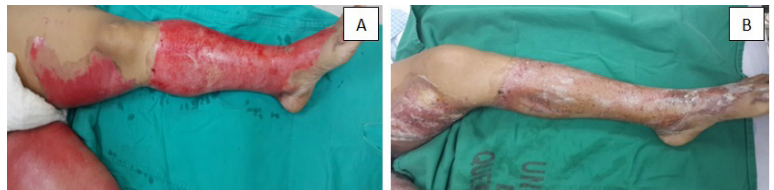

Figura 4A. Preoperatorio de lesión en miembro inferior izquierdo por líquido caliente. B. Postoperatorio de 10 días. 
RESULTADOS

Con el análisis de los datos obtenidos se determinó una población de 14 pacientes, todos con quemaduras de segundo grado superficial hasta $25 \%$ de superficie corporal quemada, a los que se les colocó amnios por única ocasión; 9 del sexo masculino (64\%) y 5 del femenino (36\%), con un rango de edades entre 25 y 86 años. La media fue 45 años y mediana de 47 años, las causas de las lesiones fueron: 9 casos por flama (64\%), 4 por líquido caliente ( $28 \%$ ), 1 por electricidad $(8 \%)$; las regiones anatómicas que se cubrieron fueron: 4 pacientes en miembros superiores y tórax anterior ( $28 \%), 2$ exclusivamente en tórax anterior $(16 \%)$, 4 solo en miembros superiores $(28 \%), 4$ en miembros inferiores $(28 \%)$ como se observa en la (tabla).

TABLA. DATOS DEMOGRÁFICOS, CLÍNICOS Y CAUSA DE LA QUEMADURA DE PACIENTES EN ESTUDIO

\begin{tabular}{|c|c|c|c|c|}
\hline $\begin{array}{l}\text { EDAD } \\
\text { AÑOS }\end{array}$ & SEXO & $\begin{array}{l}\text { DÍAS POSO- } \\
\text { PERATORIOS }\end{array}$ & CAUSA & $\begin{array}{l}\text { REGIONES } \\
\text { ANATÓMICAS }\end{array}$ \\
\hline 58 & $\mathrm{~F}$ & 13 & Líquido caliente & Tórax \\
\hline 56 & M & 6 & $\begin{array}{l}\text { Flama por fuga de } \\
\text { gas doméstico }\end{array}$ & $\begin{array}{l}\text { Miembro superior } \\
\text { derecho e izquierdo }\end{array}$ \\
\hline 25 & M & 6 & $\begin{array}{l}\text { Flama por fuga de } \\
\text { gas doméstico }\end{array}$ & $\begin{array}{l}\text { Miembro superior } \\
\text { derecho e izquierdo }\end{array}$ \\
\hline 46 & M & 6 & $\begin{array}{l}\text { Electricidad cable } \\
\text { alto }\end{array}$ & $\begin{array}{l}\text { Miembro superior } \\
\text { derecho }\end{array}$ \\
\hline 31 & M & 12 & Líquido caliente & Tórax \\
\hline 51 & M & 12 & Líquido caliente & $\begin{array}{l}\text { Miembro inferior } \\
\text { derecho }\end{array}$ \\
\hline 32 & $\mathrm{~F}$ & 9 & $\begin{array}{l}\text { Flama por fuga de } \\
\text { gas doméstico }\end{array}$ & $\begin{array}{l}\text { Miembro inferior } \\
\text { derecho e izquierdo }\end{array}$ \\
\hline 86 & M & 13 & $\begin{array}{l}\text { Flama por fuga de } \\
\text { gas industrial }\end{array}$ & $\begin{array}{l}\text { Miembro superior } \\
\text { derecho y tórax }\end{array}$ \\
\hline 36 & $M$ & 10 & $\begin{array}{l}\text { Flama por tanque } \\
\text { de brea }\end{array}$ & $\begin{array}{l}\text { Miembro superior } \\
\text { derecho y tórax }\end{array}$ \\
\hline 28 & $\mathrm{~F}$ & 13 & $\begin{array}{l}\text { Flama por fuga de } \\
\text { gas doméstico }\end{array}$ & $\begin{array}{l}\text { Miembros superiores } \\
\text { y tórax }\end{array}$ \\
\hline 32 & $\mathrm{~F}$ & 6 & $\begin{array}{l}\text { Flama por fuga de } \\
\text { gas doméstico }\end{array}$ & $\begin{array}{l}\text { Miembros superiores } \\
\text { y tórax }\end{array}$ \\
\hline 51 & M & 6 & Líquido caliente & $\begin{array}{l}\text { Miembro inferior } \\
\text { derecho }\end{array}$ \\
\hline 47 & $\mathrm{~F}$ & 11 & $\begin{array}{l}\text { Flama por fuga de } \\
\text { gas doméstico }\end{array}$ & Miembros inferiores \\
\hline 61 & M & 12 & $\begin{array}{l}\text { Flama por fuga de } \\
\text { gas doméstico }\end{array}$ & Miembros superiores \\
\hline
\end{tabular}

El promedio de estancia hospitalaria postoperatoria fue de 9 días, con un límite inferior de 6 y superior de 13. En ningún paciente se observó signos de infección o rechazo inmunológico y en todos los casos se observó reepitelización de las lesiones, en la escala de valoración numérica del dolor (del 1 al 10), se confirmó que todos los pacientes referían menos dolor y posteriormente al día 7 desapareció en todos los pacientes (figura 5).

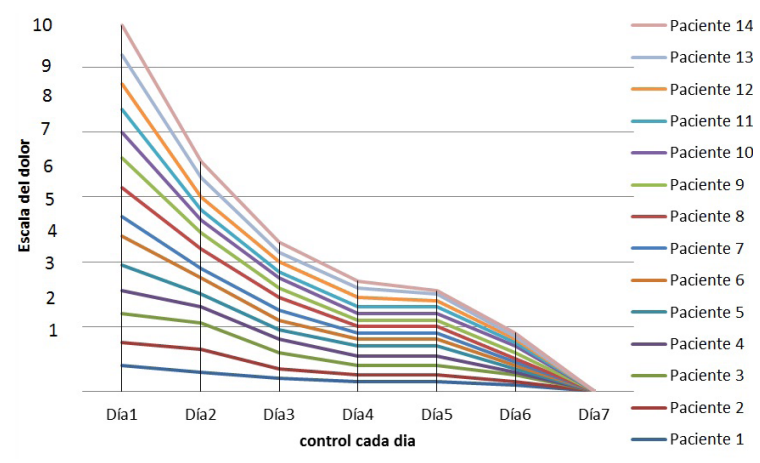

Figura 5. Escala de valoración numérica del dolor del 1 al 10 , se observa notable disminución de la intensidad del dolor al día 4 del postoperatorio.

\section{DISCUSIÓN}

El uso de membrana amniótica ha sido reportado en varias patologías relacionadas a diversos campos: en lesiones de la superficie del globo ocular en oftalmología, en ginecología para reconstrucciones vaginales, en neonatología para reparaciones de onfalocele y en cirugía plástica, especialmente en pacientes quemados, para cobertura transitoria de grandes áreas cruentas ${ }^{14}$. Las características histológicas y biofísicas del amnios permite ser aplicado a los defectos ya mencionados, favoreciendo la epitelización.

Al inhibir las proteasas brinda un efecto antiinflamatorio directo sobre las heridas mejorando su aspecto y evolución. Además, evita la pérdida de líquidos, electrolitos y proteínas en las áreas cruentas, siendo de gran ayuda en pacientes quemados; a modo de barrera artificial ayuda a prevenir las infecciones como una membrana basal sustituta, y tiene un importante efecto analgésico sobre las lesiones.

Procede confirmar que todas estas propiedades han sido observadas en los pacientes; en esta serie de casos se documentó de manera muy evidente el efecto analgésico y mejoría de la evolución de las heridas.

La constitución del epitelio de la $\mathrm{MAH}$ permite múltiples funciones especializadas, su membrana basal, contiene colágeno tipo IV y $\mathrm{V}$, laminina e integrina, las cuales actúan en procesos ligados a la cicatrización e inflamatorios. La matriz estromal está conformada en su mayoría por fibroblastos, agentes celulares involucrados directamente en procesos de re- 
paración y cicatrización de heridas. Inmediato a su uso como cobertura en heridas, facilita la migración epitelial, refuerza su adherencia, promueve la diferenciación celular y previene la apoptosis.

Un mecanismo de relevancia es la protección física que adquiere el tejido cruento con el uso de la $\mathrm{MAH}$, proporcionando cobertura ante cualquier acción física de barrido en contra de la piel lesionada. ${ }^{15}$ Este método funciona como una barrera entre la herida y el medio externo siendo una de sus causas de fracaso: su uso sobre tejido desvitalizado o necrótico. Se recomienda que las superficies contaminadas e infectadas deben ser desinfectadas previo a su colocación. ${ }^{16}$

Los resultados del uso del amnios fueron marcadamente favorables para los pacientes de este estudio; por lo tanto podemos concluir que este método de cobertura temporal de heridas, es eficaz y se sugiere su aplicación en casos necesarios como una alternativa para tratamiento de superficies cruentas las causadas por quemaduras.

\section{REFERENCIAS BIBLIOGRÁFICAS}

1. Sawhney CP. Amniotic membrane as a biological dressing in the management of burns. Burns 1989; 15: 339-42

2. Meller D, Pires RT, Mack RJ, Figueiredo F, Heiligenhaus A, Park WC et al. Amniotic membrane transplantation for acute chemical or thermal burns. Ophthalmology 2000; 107: 980-990.

3. Sippel Kimberly C. Amniotic membrane surgery. Current opinion in Ophthalmology 2001; 12(4): 269-28.

4. Davis JW. Skin transplantation with a review of 550 cases at Johns Hopkins Hospital. Johns Hopkins Med. J. 1910, 15:307
5. Stern M. The grafting of preserved amniotic membrane to burned and ulcerated surfaces, substituting skin grafts. JAMA 1913, 60:973

6. Sabella N. Use of the fetal membrana in skin grafting Med. Rec. NY 1913, 83:478

7. De Roth A. Plastic repair of conjuntival defects with fetal membrana . Arch. Ophthalmol 1940, 23:522-5

8. Li DQ, Lee SB, Gunja-Smith Z, et al. Over expression of collagenase (MMP-1) and stromelysin (MMP-3) by pterigium head fibroblasts. Arch. Ophthalmol 2001, 119:71-80

9. Fukuda K, Chikama T, Nakamura M, Nishida $T$. Differential distribution of subchains of the basement membrane components type IV collagen and laminin among the amniotic membrane, cornea, and conjunctiva. Cornea 1999; 18: 73-79.

10. Boudreau N, Sympson CJ, Werb Z et al. Suppression of ICE and apoptosis in mammary epithelial cells by extracellular matrix. Science 1995; 267: 891-893.

11. Hurme $M$, Lahdenpohja $N$, Santtila S. Gene polymorphisms of interleukins 1 and 10 in infectious and autoimmune diseases. Ann Med 1998; 30: 469-73

12. Sistani F, Erb C, Brockmann D et al. Human amniotic membrane after different cryopreservation methods. Invest Ophthalmol Vis Sci 2001; 42: S269.

13. Koizumi N, Fullwood NJ, Bairaktaris G et al. Cultivation of corneal epithelial cells on intact and denuded human amniotic membrane. Invest Ophthalmol Vis Sci 2000; 41: 2506-2513.

14. Cerizola M, Fossati G. Diferentes aplicaciones del amnios en cirugía. Cir Urug 1984; 54:224-9

15. Van Herendael BJ, Oberti C, Brosens I. Microanatomy of the human amniotic membranes. A light microscopic, transmission, and scanning electron microscopic study. Am J Obstet Gynecol 1978; 131: 872-880.

16. Choi, Tae Hoon. Antiinflammatory effects of amniotic membrane transplantation in ocular surface disorders cornea 2001; 20(4): 408-413. 